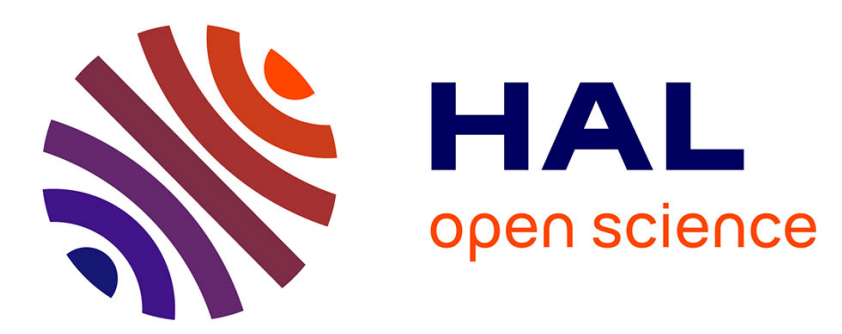

\title{
A perception-inspired building index for automatic built-up area detection in high-resolution satellite images
}

Gang Liu, Gui-Song Xia, Xin Huang, Wen Yang, Liangpei Zhang

\section{To cite this version:}

Gang Liu, Gui-Song Xia, Xin Huang, Wen Yang, Liangpei Zhang. A perception-inspired building index for automatic built-up area detection in high-resolution satellite images. IGARSS'2013, Jul 2013, Australia. pp.1-4. hal-00821316

\section{HAL Id: hal-00821316 https://hal.science/hal-00821316}

Submitted on 9 May 2013

HAL is a multi-disciplinary open access archive for the deposit and dissemination of scientific research documents, whether they are published or not. The documents may come from teaching and research institutions in France or abroad, or from public or private research centers.
L'archive ouverte pluridisciplinaire HAL, est destinée au dépôt et à la diffusion de documents scientifiques de niveau recherche, publiés ou non, émanant des établissements d'enseignement et de recherche français ou étrangers, des laboratoires publics ou privés. 


\title{
A PERCEPTION-INSPIRED BUILDING INDEX FOR AUTOMATIC BUILT-UP AREA DETECTION IN HIGH-RESOLUTION SATELLITE IMAGES
}

\author{
Gang Liu ${ }^{1,2}$, Gui-Song Xia ${ }^{1}$, Xin Huang ${ }^{1}$, Wen Yang ${ }^{1,2}$, Liangpei Zhang ${ }^{1}$ \\ ${ }^{1}$ State Key Lab. of Information Engineering in Surveying, Mapping and Remote Sensing, \\ LIESMARS, Wuhan University, Wuhan, China \\ ${ }^{2}$ Signal Processing Lab., Electronic Information School, \\ Wuhan University, Wuhan, China
}

\begin{abstract}
This paper addresses the problem of automatic extraction of built-up areas from high-resolution remote sensing images. We propose a new building presence index from the point view of perception. We argue that built-up areas usually result in significant corners and junctions in high-resolution satellite images, due to the man-made structures and occlusion, and thus can be measured by the geometrical structures they contained. More precisely, we first detect corners and junctions by relying on a perception-inspired corner detector, called an a-contrario junction detector. Each detected corner is associated with a perceptual significance, which measures the structural saliency of the corner in the image and is independent of the contrast and scale. All these detected corners together with their significance are then used to compute the building index. The proposed approach is evaluated on a high-resolution satellite image set, including 15 big images from GeoEye-1, QuickBird and IKONOS. The results demonstrated that our method achieves the state-of-the-art results and can be used in practical applications.
\end{abstract}

Index Terms - High-resolution satellite images, junction detection, built-up area detection, contrast independent, urban area detection.

\section{INTRODUCTION}

The detection of built-up areas is a dynamic and challenging problem in remote sensing. High resolution satellite images provide rich information and enable accurate locating of built-up areas. However, manual administration of these areas from huge images is time consuming and unfeasible, thus automatic approaches are demanded to detect the built-up area from aerial and satellite images.

In the last decade, a large number of automatic built-up area detection approaches have been reported [1, 2, 3, 4, 5, 6, 7, 8], which can be roughly divided into two categories. The first category of such methods rely on supervised classification. More precisely, they use a large set of specific training samples to learn the feature distribution of built-up areas for de- tection, see $[1,2]$. The second category of methods directly detect built-up areas without using any training data. It includes the approaches using local key point features [3, 4, 7], contrast measure of texture features (e.g. PanTex using the gray-level co-occurrence matrix [6]) and the difference of morphological profiles [8].

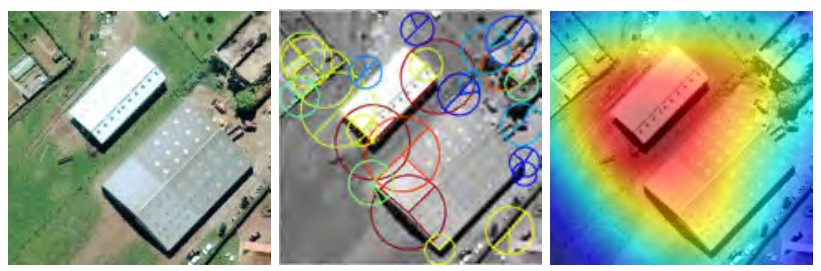

Fig. 1: From left to right: a small GeoEye image containing buildings, junctions detected by ACJ, and computed PBI. The color indicates the significant level of buildings (red corresponds to hight level).

This paper addresses the problem of unsupervised automatic extraction of built-up areas, inspired by a perception guided approach, named an a-contrario junction (ACJ) detector $[9,10]$, which extracts significant and meaningful geometric structures in natural images and is robust to textures. The main idea is that built-up areas usually result in significant geometric structures (e.g. corners and junctions) in highresolution satellite images, due to their man-made characteristics and occlusions, and thus can be indexed and measured by the local geometrical structures they contained. More precisely, given an image, we first apply the ACJ detector to find all perceptually significant geometrical structures, each of which is associated with a significance measuring its saliency in the image. The significance of local structures is then propagated to the whole image by using a spatial kernel, in order to compute a built-up area presence index, named perceptual building index (PBI) for short. It is worth noticing that the $\mathrm{PBI}$ is independent of local image contrast, meaning that it detects white and black built-up areas equivalently. As we shall see in Section 2, PBI achieves the state-of-the-art results on built-up area detection. 
In the rest of this paper, we first briefly recall the ACJ detector in Section 2, detail the computation of the perceptual building index in Section 3, then illustrate experiments in Section 4 and finally conclude in Section 5.

\section{AN A-CONTRARIO JUNCTION DETECTOR}

This section briefly recall the accurate and contrast invariant junction detector, named ACJ, originally introduced by Xia et. al. [10,9]. A junction $\mathrm{j}$ is represented by its center $\mathbf{p}$, its scale $r \in \mathbb{N}$ and a set of $M$ branch directions $\left\{\theta_{1}, \ldots \theta_{M}\right\}$ around p. $M$ is the order of the junction. We consider $M=$ 2, 3, corresponding to corners and T-(or Y-) junctions. More precisely, given a gray-scale image $I$ on discrete image lattice $\Omega$ and denote the gradient as $\nabla I=\left(I_{x}, I_{y}\right)$. We define the direction $\phi(\mathbf{q})$ of a pixel $\mathbf{q}$ as the direction perpendicular to the gradient at $\mathbf{q}$, i.e. $\phi(\mathbf{q})=\left(\arctan \frac{I_{y}(\mathbf{q})}{I_{x}(\mathbf{q})}+\frac{\pi}{2}\right) \bmod (2 \pi)$.

The ACJ detector relies on the a-contrario detection theory [11], who states that meaningful events are those whose probabilities of occurrence under some null hypothesis $\mathcal{H}_{0}$ is small enough. In the context of junction detection, the null hypothesis is defined as follows. Denoting I a random image, $\|\widetilde{\nabla \mathbf{I}}(\mathbf{q})\|$ and $\phi_{\mathbf{I}}(\mathbf{q})$ the random variables corresponding to the contrast and orientation at each pixel $\mathbf{q}$, we say that these variables follow the null hypothesis $\mathcal{H}_{0}$ if (1) $\forall \mathbf{q} \in \Omega$, $\|\widetilde{\nabla \mathbf{I}}(\mathbf{q})\|$ follows a Rayleigh distribution with parameter 1 ; (2) $\forall \mathbf{q} \in \Omega, \phi_{\mathbf{I}}(\mathbf{q})$ is uniformly distributed over $[0,2 \pi]$; (3) the family $\left\{\|\widetilde{\nabla \mathbf{I}}(\mathbf{q})\|, \phi_{\mathbf{I}}(\mathbf{q})\right\}_{\mathbf{q} \in \Omega}$ is made up of independent random variables.

Let $\jmath$ be a junction in a random image $\mathbf{I}$ where normalized gradients and directions are assumed to follow $\mathcal{H}_{0}$. The strength of the junction $y$ is defined as

$$
t(\jmath):=\min _{m=1 \ldots M} \omega_{\mathbf{p}}\left(r, \theta_{m}\right),
$$

where $\omega$ is the strength of a branch $S_{\mathbf{p}}(r, \theta)$ as $\omega_{\mathbf{p}}(r, \theta)=$ $\sum_{\mathbf{q} \in S_{\mathbf{p}}(r, \theta)} \gamma_{\mathbf{p}}(\mathbf{q})$, and $\gamma_{\mathbf{p}}(\mathbf{q})=\|\widetilde{\nabla I}(\mathbf{q})\| \cdot(\mid \cos (\phi(\mathbf{q})-$ $\alpha(\overrightarrow{\mathbf{p q}}))|-| \sin (\phi(\mathbf{q})-\alpha(\overrightarrow{\mathbf{p q}})) \mid)$.

Under the hypothesis $\mathcal{H}_{0}$, the probability that the random variable $\mathbf{t}(\jmath)$ is larger than a given threshold $t$ finally is

$$
F_{\jmath}(t):=\mathbb{P}_{\mathcal{H}_{0}}[\mathbf{t}(\jmath) \geq t]=\prod_{m=1}^{M} \int_{t}^{+\infty} \star_{j=1}^{J\left(r, \theta_{m}\right)} \mu(d z),
$$

where $J\left(r, \theta_{m}\right)$ is the size of a sector of orientation $\theta_{m}$ at scale $r$.

Each junction $\jmath$ is associated with a significant level called number of false alarms (NFA)

$$
\operatorname{NFA}(\jmath):=\# \mathcal{J}(M) \cdot F_{\jmath}(t(\jmath)),
$$

where $\# \mathcal{J}(M)$ is the number of test in an image. Observe that the smaller the NFA is, the more meaningful the junction ر, thus we use $-\log (\mathrm{NFA})$ as the significance of the junction.

Notice that, in practice, a single junction can be detected at multiple scales and multiple locations with slight differences.
These redundancies are removed by relying on an exclusion principle, called maximality. A simple example is shown in Figure 1.

For more details about the ACJ detector, please refer to [9].

\section{PERCEPTUAL BULIT-UP INDEX}

We argue that around junction structures, especially corners and T-junctions, a built-up area can be found with a high probability. Let $J:\left\{\jmath, \jmath=\left\{\mathbf{q}, r,\left\{\theta_{m}\right\}\right\}\right\}$ be the list of detected junctions in a gray-level image $I$. We define the built-up area presence index, i.e. perceptual building index (PBI), of a pixel $\mathbf{p} \in \Omega$ in the image $I$, as

$$
\operatorname{PBI}(\mathbf{p})=\sum_{\mathbf{q}_{\jmath}, \jmath \in J}-\log (\operatorname{NFA}(\jmath)) \cdot G_{r}\left(\mathbf{p}, \mathbf{q}_{\jmath}\right),
$$

where $G_{\sigma}(\mathbf{p}, \mathbf{q})=e^{-\frac{\|\mathbf{p}-\mathbf{q}\|_{2}^{2}}{2 \sigma^{2}}}$ is a Gaussian kernel with size of $\sigma$, in order to propagate the saliency of the junction $\jmath$ at $\mathbf{q}$ to the pixels $\mathbf{p}$ around $\mathbf{q}$. Observe that this propagation kernel is similar to that in [7], called spatial voting, where $\sigma$ is estimated by using morphological operations. However in our case, we simply take $\sigma=5 r$, with $r$ as the scale of junction.

To obtain a detection map of the built-up areas, we can threshold the PBI values. For instance, in this paper, we simply take the threshold as the mean of the PBI in the image. One could also use an adaptive threshold, similar to [7]. See Figure 1 for an example.

\section{EXPERIMENTAL ANALYSIS}

In order to evaluate our method, we test the proposed PBI on a set of high-resolution satellite images, including 5 GeoEye1 images, 5 IKONOS images and 5 QuickBird images. The smallest image is with size of $2000 \times 3000$ pixels.

Figure 2 shows two GeoEye-1 images in the testing set. Figure 3 compares the results of PBI with those of two stateof-the-art methods, i.e. the Modified Harris for Edges and Corners (MHEC) [7] and the morphological building index (MBI) [8]. In Figure 3, the color indicates the confident level of built-up areas, with red corresponding to high level and blue corresponding to low level. Notice that the proposed PBI avoids non-urban textures and focuses more on built-up areas, comparing with the other two approaches. In order to achieve a detection of built-up areas, a threshold is applied to the PBI indexes. For PBI, the threshold is set to be the mean of the PBI values in each image. While the parameters of MHEC and $\mathrm{MBI}$ are selected according to their original references [7] and [8]. One can observe that the detection results by PBI are with less false alarms and more correct detections. Figure 3 demonstrates similar results on the GeoEye-1 France image. Both MHEC and MBI have heavy responses in texture areas. It is mainly because MHEC relies on edge information and 

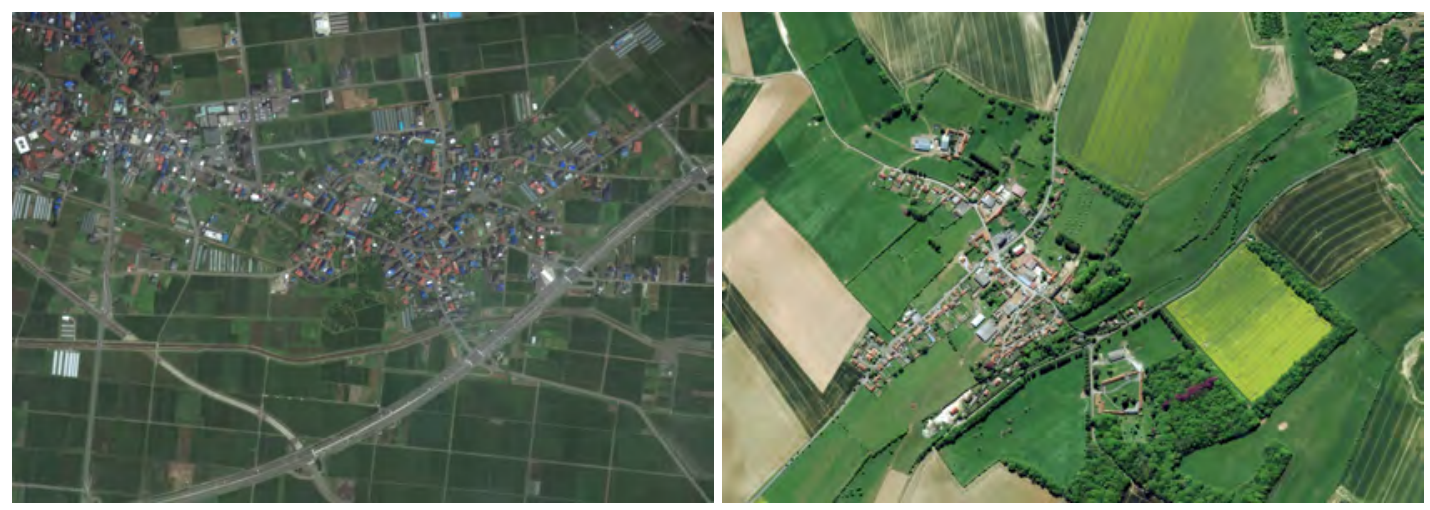

Fig. 2: Two high-resolution satellite images in the testing set. Left: GeoEye-1 images at Inakadate, with size of $3000 \times 2200$ pixels; Right: GeoEye-1 images in France, with size of $3200 \times 2300$.

MBI use texture features. More results and the full-resolution version of the images in this paper can be found at http: / / dsp.whu. edu.cn/cn/staff/yw/PBI.html.

It is also worth noticing that the running time of the PBI on a $3000 \times 2000$ image is about 5 seconds, which is much less that those of MHEC and MBI.

\section{CONCLUSION}

This paper has proposed a perception-inspired building presence index for built-up area extraction in high-resolution satellite images. It is based on a probabilistic perception theory (i.e. a-contrario methodology) and benefits from a robust and contrast invariant junction detector (i.e. the ACJ detector). Moreover, the proposed method is free of parameter and fully automatic. Although the experimental results demonstrate that PBI achieves the state-of-the-art results on built-up area detection, a potential extension is to take into account more powerful perception rules, such as parallel lines. The fast computation of PBI also enables us to use it for practical applications.

\section{REFERENCES}

[1] J.A. Benediktsson, M. Pesaresi, and K. Amason, "Classification and feature extraction for remote sensing images from urban areas based on morphological transformations," IEEE Trans. on Geoscience and Remote Sensing, vol. 41, no. 9, pp. 1940 - 1949, sept. 2003.

[2] Ping Zhong and Runsheng Wang, "A multiple conditional random fields ensemble model for urban area detection in remote sensing optical images," IEEE Trans. on Geoscience and Remote Sensing, vol. 45, no. 12, pp. 3978 -3988, dec. 2007.

[3] B. Sirmacek and C. Unsalan, "Urban-area and building detection using sift keypoints and graph theory," Geo- science and Remote Sensing, IEEE Transactions on, vol. 47, no. 4, pp. 1156 -1167, april 2009.

[4] B. Sirmacek and C. Unsalan, "Urban area detection using local feature points and spatial voting," Geoscience and Remote Sensing Letters, IEEE, vol. 7, no. 1, pp. 146 -150 , jan. 2010.

[5] B. Sirmacek and C. Unsalan, "A probabilistic framework to detect buildings in aerial and satellite images," IEEE Trans. on Geoscience and Remote Sensing, vol. 49, no. 1, pp. $211-221$, jan. 2011.

[6] M. Pesaresi, A. Gerhardinger, and F. Kayitakire, "A robust built-up area presence index by anisotropic rotation-invariant textural measure," Selected Topics in Applied Earth Observations and Remote Sensing, IEEE Journal of, vol. 1, no. 3, pp. $180-192$, sept. 2008.

[7] A. Kovacs and T. Sziranyi, "Improved harris feature point set for orientation-sensitive urban-area detection in aerial images," Geoscience and Remote Sensing Letters, IEEE, vol. PP, no. 99, pp. 1 -5, 2012.

[8] Xin Huang and Liangpei Zhang, "Morphological building/shadow index for building extraction from highresolution imagery over urban areas," Selected Topics in Applied Earth Observations and Remote Sensing, IEEE Journal of, vol. 5, no. 1, pp. 161 -172, feb. 2012.

[9] G.-S. Xia, J. Delon, and Y. Gousseau, "Accurate junction detection and characterization in natural images," Tech. Rep., HAL-preprint-00631609, 2011.

[10] G.-S. Xia, J. Delon, and Y. Gousseau, "An accurate and contrast invariant junction detector," in International Conference of Pattern Recognition (ICPR), 2012.

[11] A. Desolneux, L. Moisan, and J.-M. Morel, "Meaningful alignments," IJCV, vol. 40, no. 1, pp. 7-23, 2000. 


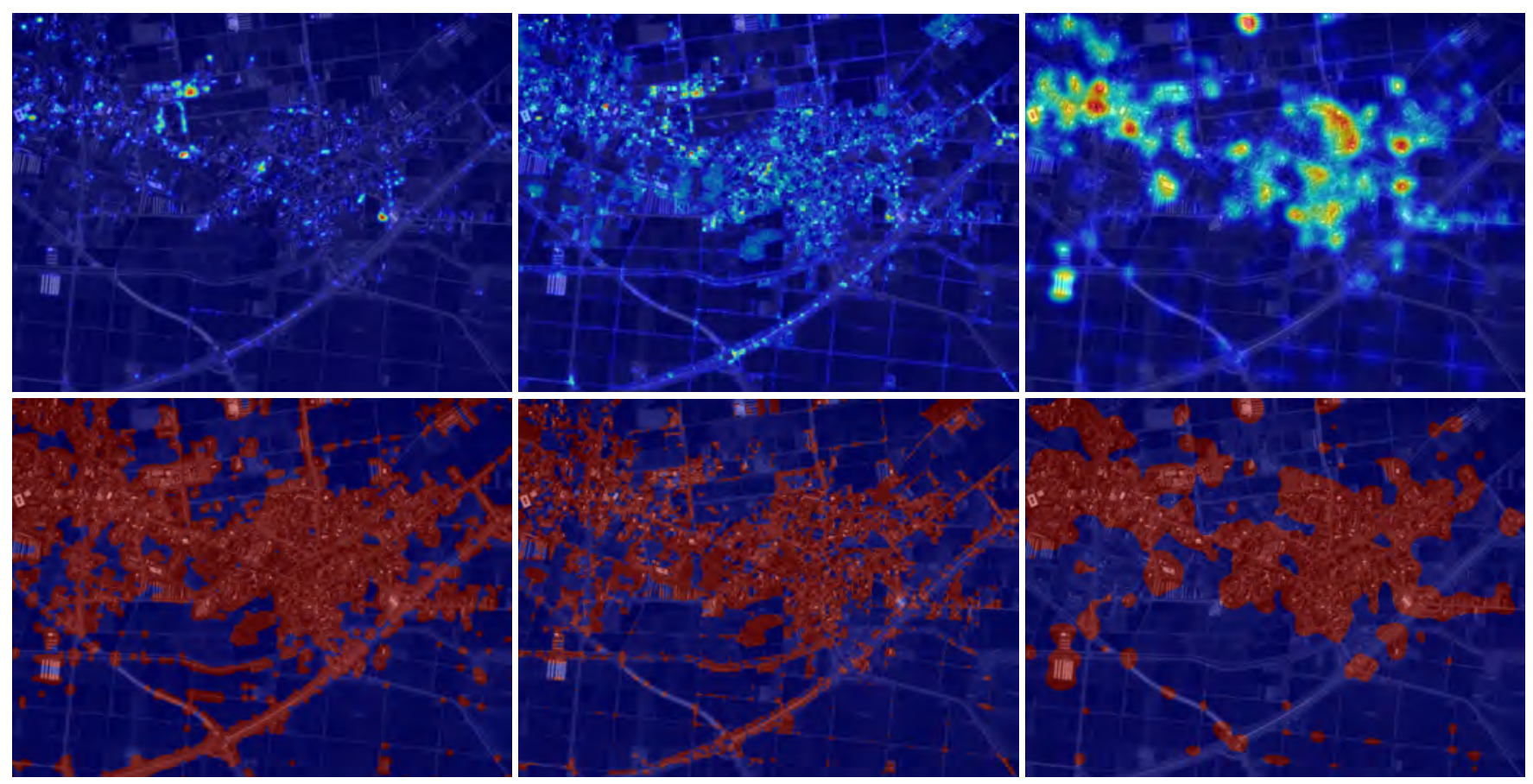

Fig. 3: The results of proposed perceptual building index (PBI) and detection of built-up areas on the GeoEye-1 France image. From left to right are the results of $\mathrm{MHEC}$ [7], $\mathrm{MBI}$ [8] and PBI, respectively. The top row shows the PBI results and the bottom row displays the detected built-up areas. In the PBI map, the color indicates the confident level of built-up areas, with red corresponding to high level and blue corresponding to low one.
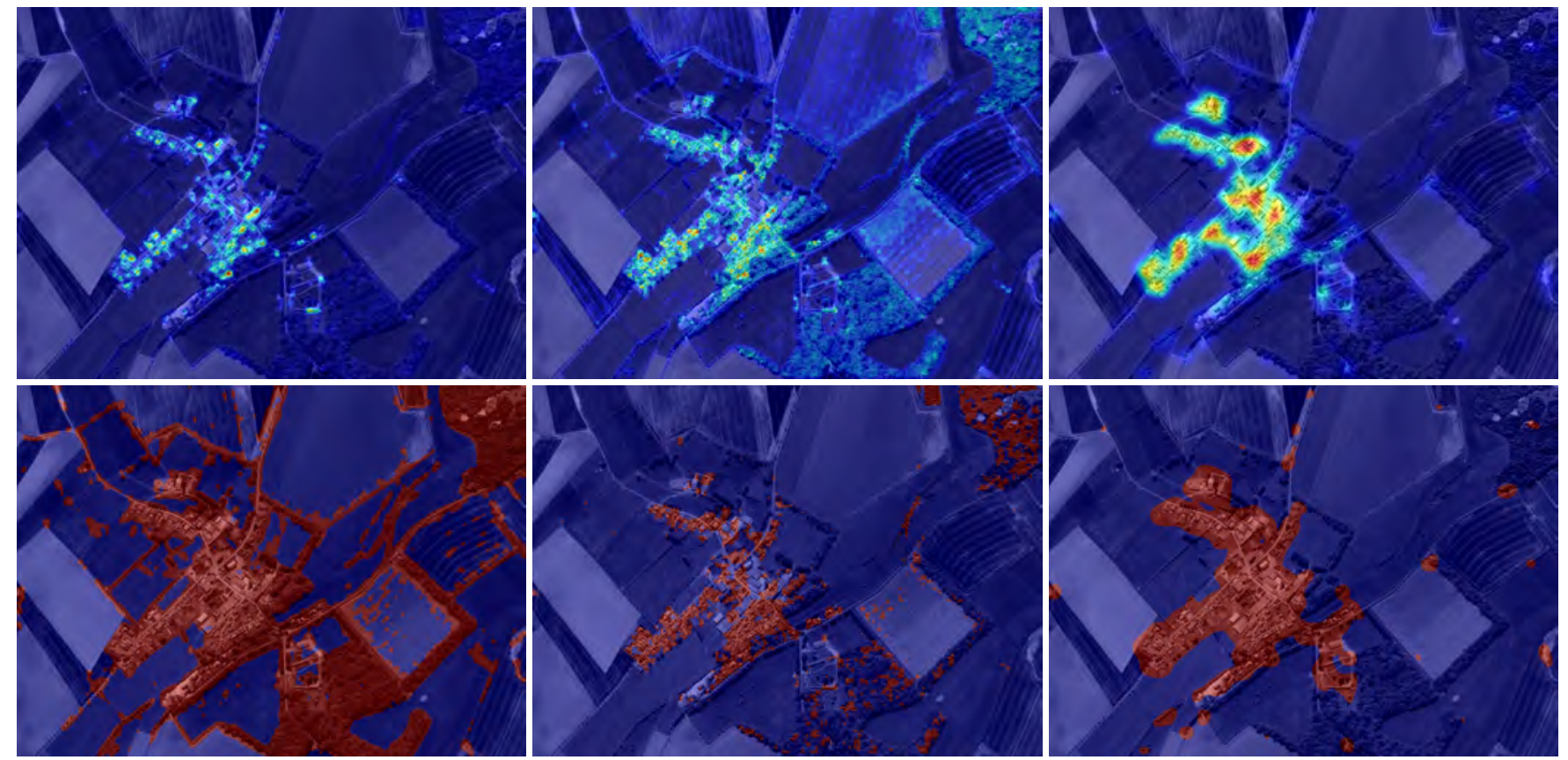

Fig. 4: The results of proposed perceptual building index (PBI) and detection of built-up areas on the GeoEye-1 Japan image. From left to right are the results of $\mathrm{MHEC}$ [7], $\mathrm{MBI}[8]$ and $\mathrm{PBI}$, respectively. The top row shows the PBI results and the bottom row displays the detected built-up areas. In the PBI map, the color indicates the confident level of built-up areas, with red corresponding to high level and blue corresponding to low one. 